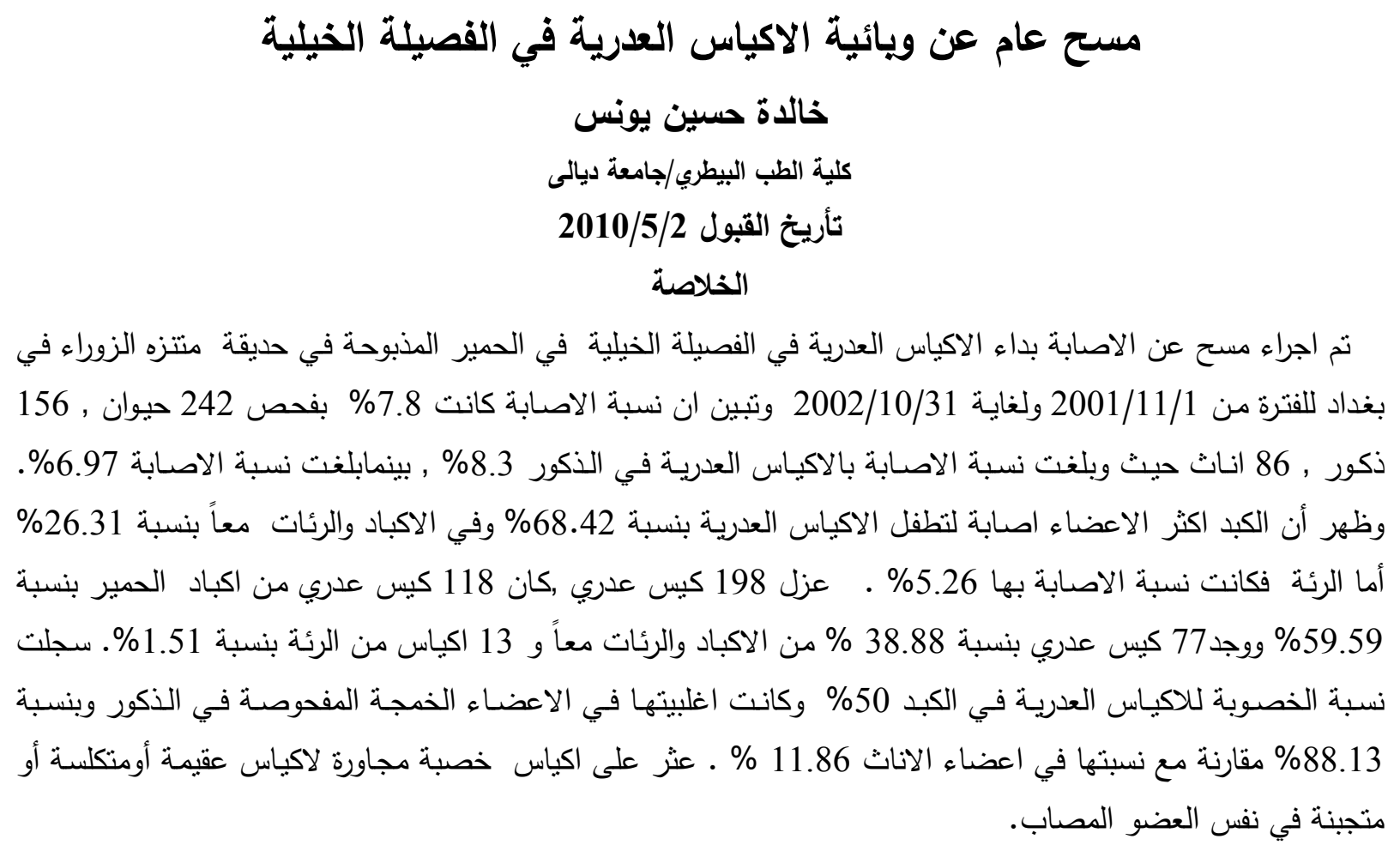

\title{
General survey on the epidemic hydatidosis in Equidae
}

\author{
Khalida Hussain Younis \\ College of Vet. Med. Diayala University.
}

\section{Summary}

A general survey was implemented on the hydatid cyst disease in Equidae duing the period from $1 \backslash 11 \backslash 2001$ till $31 \backslash 10 \backslash 2002$. The results showed that the infection rate was $7.8 \%$ by examining 242 animals, 156 males and 86 females. The rate of hydatid cysts infection reached $8.3 \%$ in males and6.97\% in females. The livers were the most infected organ by parasitic rate of $68.42 \%$,in livers and lungs together was $26.31 \%$ while in lung was $5.26 \%$.

A198 hydatid cysts were isolate from donkeys livers with rate of 59.59\%, from livers and lungs with rate of $38.33 \%$ while the isolation rate from lung was $1.5 \%$.

The fertility rate of hydatid cysts in livers reached $50 \%$, most of them were in the examined infected organs reached $11.86 \%$. Fertile hydatid cysts where found near sterile or calcified or caseated cysts in the same infected organ.

\footnotetext{
المقدمة

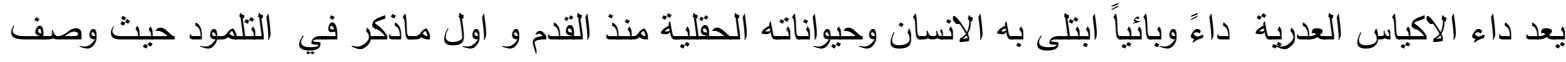

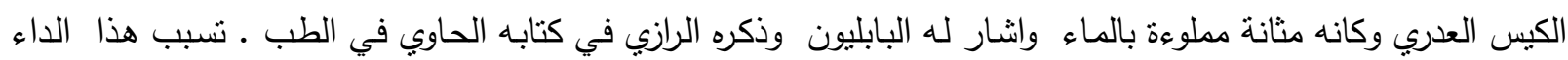

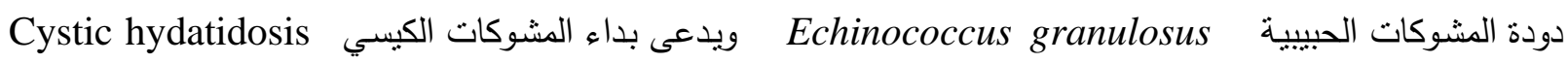
(1) • او عند اصابته افراد الفصيلة الخيلية (2و 3). تحتاج دورة حياة المشوكات الى مضيفين
} 
من اللبائن وهي اكلات اللحوم كمضيف نهائي واكلات الاعشاب والانسان كمضيف وسطي (4) ـ تعد الكلاب الخطر

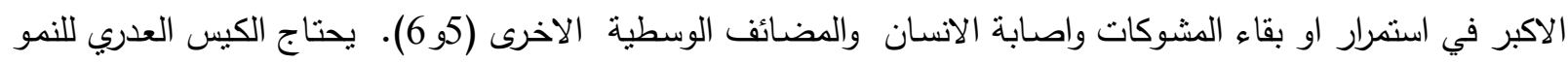

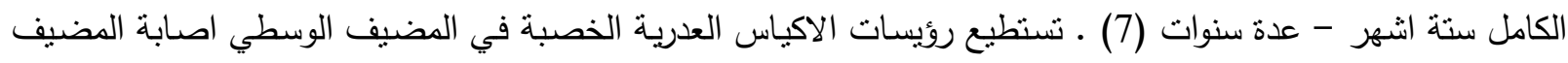

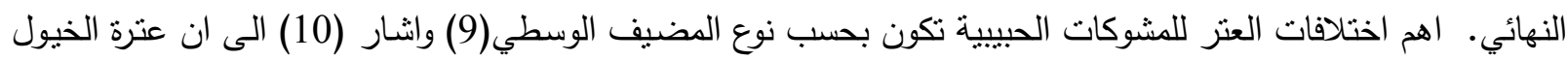
تختلف عن عترة الاغنام بايولوجياً. سجل South well عام (1927) اصابة الحمير بالاكياس العدرية في مدينة ليفربول

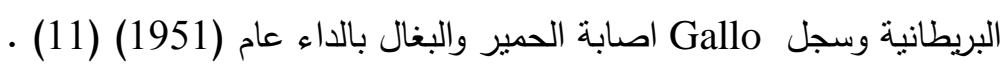

\section{المواد وطرائق العمل}

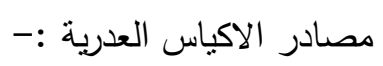

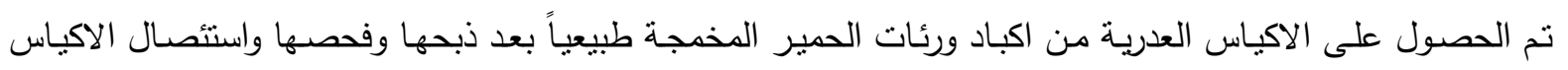

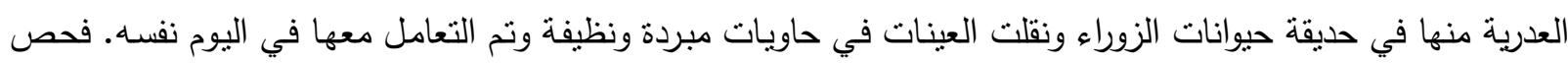

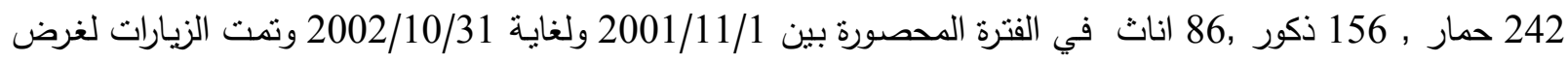

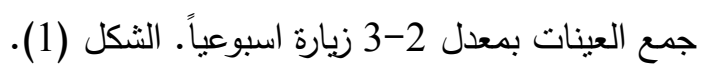

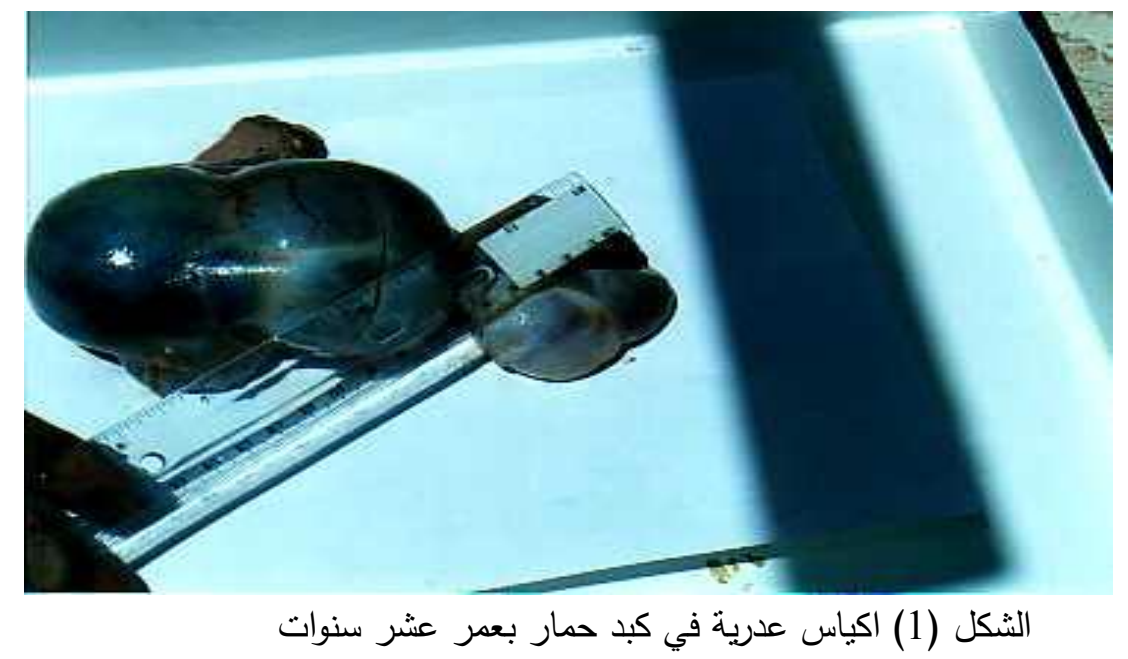

$$
\text { (2) جمع الرؤوس الاولية والسائل العدري :- }
$$

ازيلت جميع الانسجة المحيطة بالكيس العدري باستخدام الملقط والمقص والمشرط وسحبت اكبر كمية من السائل بمحاقن

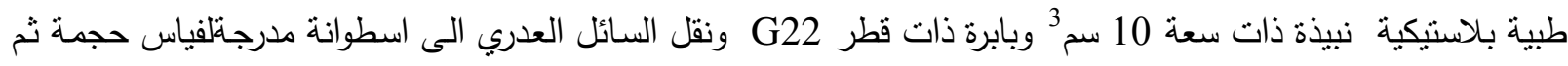

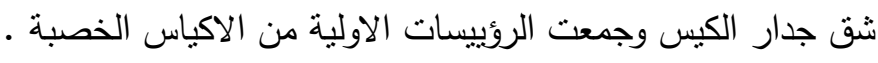


المجلة الطبية البيطرية العراقية , المجلد 34 ,العدد 1 , السنة 2010 
المجلة الطبية البيطرية العراقية , المجلد 34 ,العدد 1 , السنة 2010 
المجلة الطبية البيطرية العراقية , المجلد 34 ,العدد 1 , السنة 2010 
المجلة الطبية البيطرية العراقية , المجلد 34 ,العدد 1 , السنة 2010 


\section{النتائج}

بلغ العدد الكلي للحمير المصابة بالاكياس العدرية 19 حماراً من مجموع 242 حيوان مفحوص وبنسبة 7.85 \% ظهرت

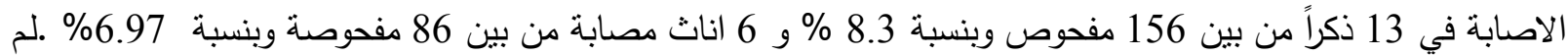
يظهر التحليل الاحصائي وجود فرق معنوي بنسبة (0.05)>p بالاصابة بداء الاكياس العدرية بين الذكور والاناث ( الجدول

سجلت اعلى الاصابات في شهر حزيران (6 حيوانات )اما في شهر كانون الثاني ونيسان فكانت 3 حيوانات مصابة لكل شهر , ووجدت اصابتين في كل من الاشهر كانون الاول وشباط وتشرين الاول واصابة واحدة في شهر اذار ولم تظهر حالة اصـابة في الاثـهر تثرين الثاني /مـايس , تموز , اب , ايلول لكل من الذكور والاناث المفحوصـة .أظهر التحليل الاحصائي وجودارتفاع بشكل معنوي 0,05 >p في الاعداد المصابة حيث كان أعلاها في حزيران مقارنة مع بقية الاشهر ووتبين أيضا وجود أرتفاع بشكل معنوي(0.05) > p في الاعداد المصابة من الذكور والاناث على التوالي وكان أعلاها في حزيران بالنسبة للذكور بينما كان أعلاها في كانون الثاني وحزيران بالنسبة للاناث مقارنة مع بقية أنشهر السنة ( الجدول 1). الجدول (1) عدد الحمير المفحوصة والمصابة بداء الاكياس العدرية لكلا الجنسين.

\begin{tabular}{|c|c|c|c|c|c|c|}
\hline العدد الكلي & العدد الكلي & عدد الذكور & اللعدد الكلي & العدد الكلي & العدد الكلي & الاشتهر \\
\hline & 12 & & 14 & & 26 & رين الثاني \\
\hline & 4 & 2 & 16 & 2 & 20 & نون الاول \\
\hline 2 & 12 & 1 & 13 & 3 & 25 & ون الثاني \\
\hline & 5 & 1 & 10 & 2 & 15 & باط \\
\hline & 9 & 1 & 5 & 1 & 14 & \\
\hline & $\overline{6}$ & 3 & 17 & 3 & 23 & سان \\
\hline & 6 & & 11 & & 17 & بس \\
\hline 2 & $\overline{7}$ & $\overline{4}$ & 18 & $\overline{76}$ & 25 & زيران \\
\hline & 7 & & 9 & & 16 & \\
\hline & 5 & & 12 & & 17 & \\
\hline & 8 & & 16 & & 24 & ايلول \\
\hline 1 & 5 & 1 & 15 & 2 & 20 & تشرين الاول \\
\hline & 86 & 13 & 156 & 19 & 242 & موع \\
\hline
\end{tabular}


الكبد هو من اكثر الاعضاء اصابة حيث استقرت الاكياس في 13 كبد مصاب وبنسبة $68.422 \%$ ( 9 ذكور و 4 اناث

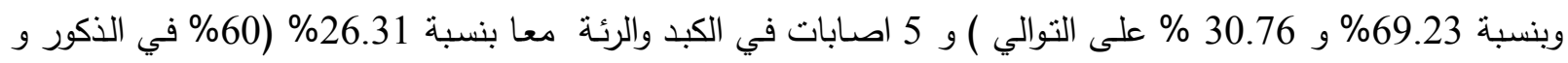
40\% في الاناث) وفي رئة حمار واحد بنسبة 5.26\% اما نسبة اصابة الاعضاء للاككور والاناث

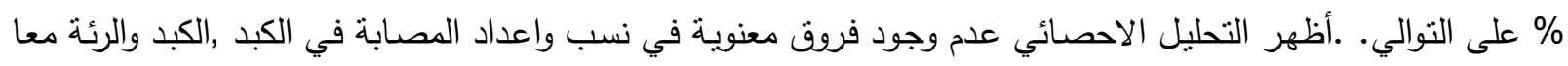

$$
\text { بين الذكور والاناث (0.05) p • (الجدول 2). }
$$

عزل 198 كيس عدري من الاعضاء المصابة ,152 في اعضاء الذكور بنسبة 76.76\% و و 46 كيس عدري في الاعضاء الاناث وبنسبة 23.23

استقر 97 كيس عدري منطفل في اكباد الذكور وبنسبة 63.81\% و 52 كيس عدري في الكبد والرئة معاً وبنسبة 34.21\%

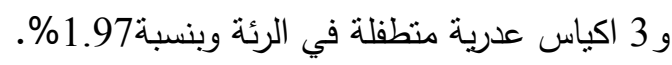

في الاناث تطفل 25 كيس عدري في الاكباد والرئات المخمجة معاً وبنسبة 54.34\% وبلغت نسبتها في الكبد 65.65 وكئه لـ 21 كيس عدري.

وجد 118 كيس عدري منطفل في الكبد وبنسبة 59.59\% و 77 كيس عدري في الكبد والرئة معاً وبنسبة 38.88\% وبنسبة 1.51 \% لتطفل 3 اكياس عدرية في الرئة.لم يظهر التحليل الاحصائي وجود فرق معنوي بنسبة (0.05) >p ( الجدول 2)

الجدول (2) عدد الحمير المفحوصة والاعضاء المصابة بداء الاكياس العدرية لكلا الجنسين

\begin{tabular}{|c|c|c|c|c|c|c|c|c|c|}
\hline الاكياس & أصابات & الاكياس & الكبئة & الاكياس & أصابات & الاكياس & الحمير & ص المفحو & الجنس \\
\hline 3 & 1 & 52 & 3 & 97 & 9 & 152 & 13 & 156 & ذكور \\
\hline & & 25 & 2 & 21 & 4 & 64 & 6 & 86 & اناث \\
\hline 3 & 1 & 77 & 5 & 118 & 13 & 198 & 19 & 242 & \\
\hline
\end{tabular}

بلغت نسبة الخصوبة الكلية للاكياس العدرية 53.03\% وكانت أغلبها في اعضاء الذكور وبنسبة 64\% . ظهر 59 كيس عدري خصب في الاكباد المصابة وبنسبة 56,19 \% (25 كيسا عدريا خصبا في الذكور و 7 كيسا في الاناث وبنسبة 88.13\% , 11.86 \% على التوالي) و 20 كيسا عدريا عقيما وبنسبة 16.94\% (18 كيسا منها في الذكور و2 في الاناث وبنسبة 90\% ,10\% على التوالي) و 22 متجبن وبنسبة 18.64\% (15 منها في الذكور

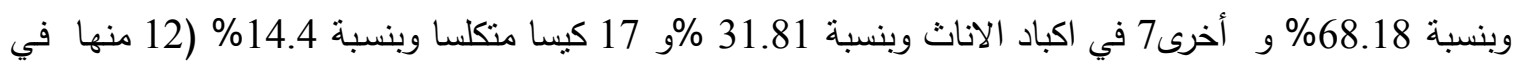
الذكور وبنسبة 70.58 \% و 5 منها بالاناث وبنسة 29.41\% ). أظهرت النتائج الاحصائيةعدم وجود فروق معنوية بين الذكور والانـاث في أعداد ونسب الاكياس العدريـة المعزولـة من الكبد الخصبة والعقيمـة والمتكلسـة والمتجبنة بمستوى (0.05) ب بن 
أظهر المسح وجود 44 كيس عدري خصب في الاكباد والرئات المخمجة معا وبنسباُ 41,9\% (26 في اكبادورئات الذكور وبنسبة 59.09\% و 18 في الاناث وبنسبة 40.90\% , 13 عقيمة بنسبة 16.88\% (8 منها في الذكور وبنسبة 61.55 \% و 5 في الاناث وبنسبة 38.46\% و 13 متجبن وبنسبة 16.88\% (12 منها وبنسبة 92.3\% وبنسبة 7.69 \% للذكور والاناث على التوالي )و 7 متكلسة وبنسبة 9.09\% ( 6 منها في الذكور وبنسبة 85.71\% و 1 في الاناث وبنسبة 14.28\%). أظهرت النتائج الاحصـائية عدم وجود فرق معنوي مهم احصـائيا بين الذكور والاناث في اعداد ونسب الاكياس العدرية الخصبة والعقيمة والمتكلسة والمتجبنة المعزولة من الكبد والرئة المصابتا معا بمستوى (0.05) الجدول (3) عدد الاكياس العدرية المعزولة من الاعضاء المصابة بالداء لكل من الذكور والاناث.

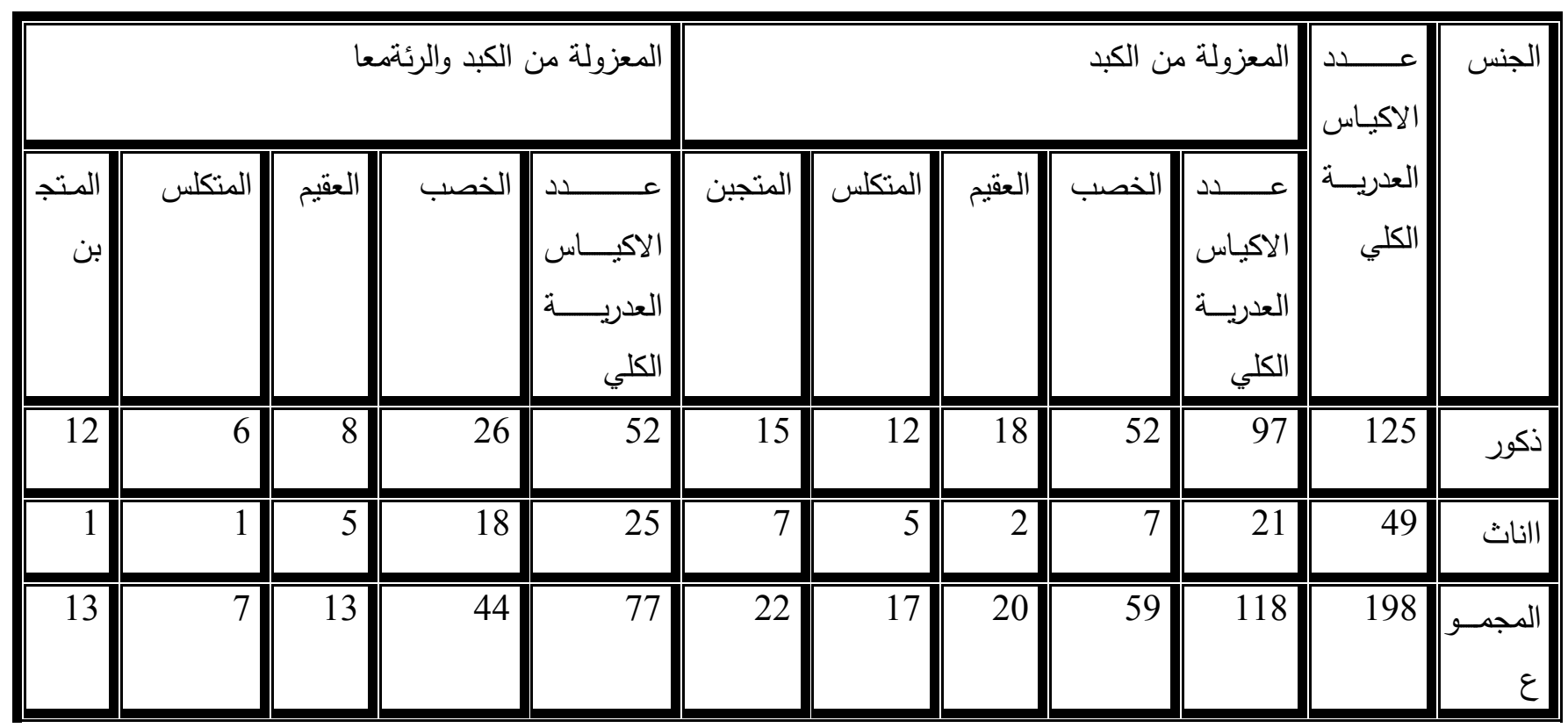

وجد كيسين خصبين في الرئة المصابة لذكر وبنسبة 66.66\% وكيس متجبن وبنسبة 33.33\%.

\section{المناقشة}

اظهرت نتائج البحث نسبة 85.85\% اصـابة الحمير بالاكياس العدريـة وهي تفوق النسبة 2.3\% التي حصل عليها بعض الباحثين (14) ومقاربة من 8.3\% التي حصل عليها البعض الاخر (15) وقد برجع السبب الى ارتفاع الاصابة في الكلاب وبالتالي ارتفاع نسبة الاصابة في المضائف الوسطية بالاضافة الى الظروف المعيشية الصعبة مما انعكس سلبا في شتى نواحي الحياة بضمنها العلف المقدم للحيوانات (وان كان رديئ النوعية) خصوصـا للحمير واستخدامها بالنقل والجر وغيرها من الاعمال المجهدة والتي تؤدي مع قلة العلف والدواء والرعاية الاخرى الى قلـة مناعـة الحيوان واصـابته بـالامراض ومنها داء الاكياس العدريـة , ذكربعض البـاحثين (16) ان انتشـار المشـوكات الحيبية وداء الاكياس العدرية يتاثز بدرجة كبيرة بطريقة اداء حيوانات المزرعة واطعام الكلاب والعادات الثـائعة

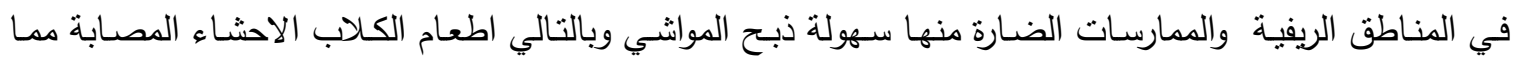
يساعد على الانتشار الواسع لداء الاكياس العدرية في المضائف الوسطية . 
فضـلا عن ذلك من العادات الثنائعة في الريف العراقي هي رمي الحيوانات النافقة على قارعة الطريق او في

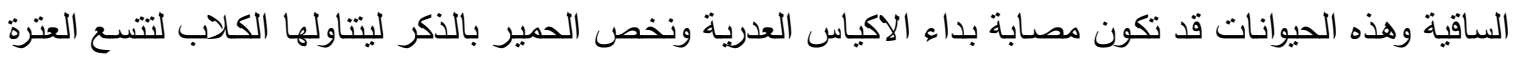

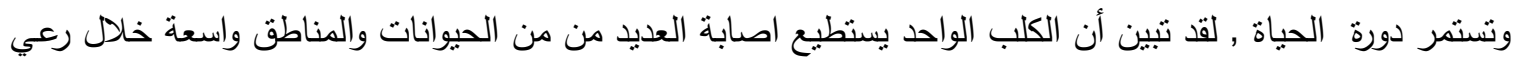

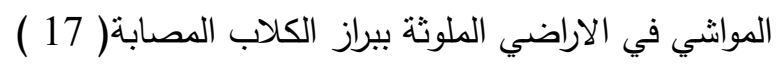

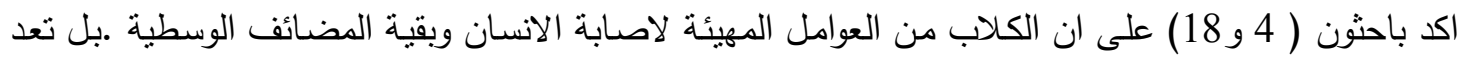

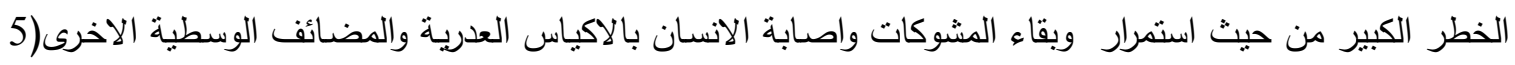

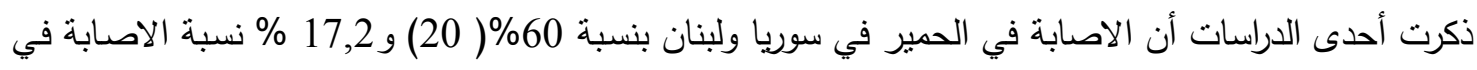

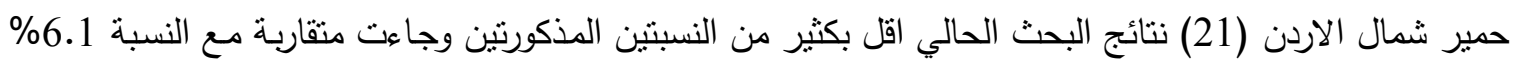

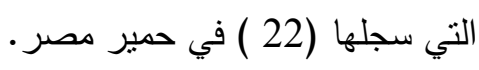

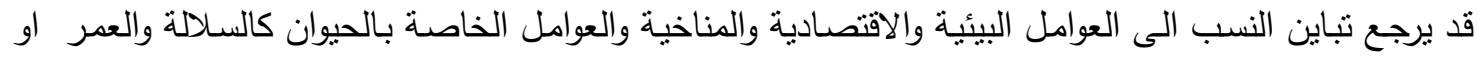

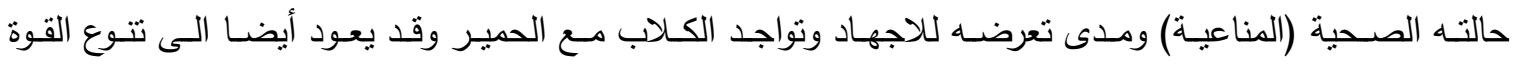

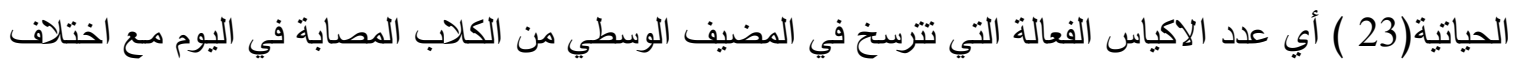
المناطق والتوزيع الجغرافي والضروف المناخية المختلفة .

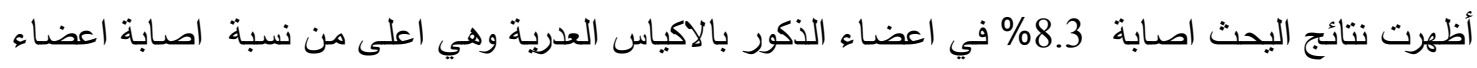

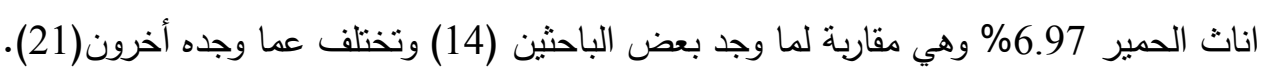

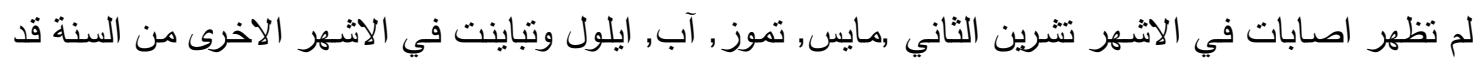

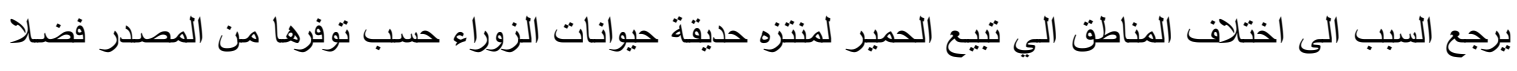

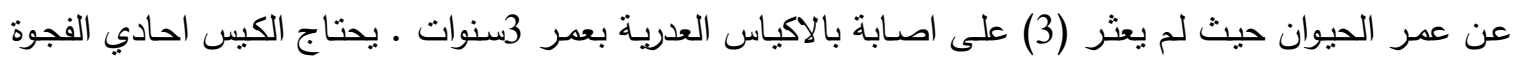
الذي ينمو بطئ عده سنوات لاكمال نموه (25,24,2) .

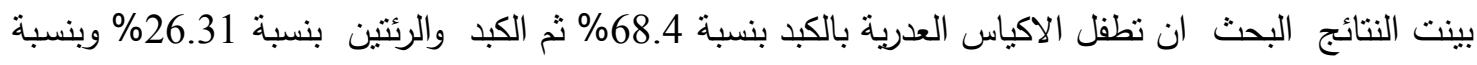

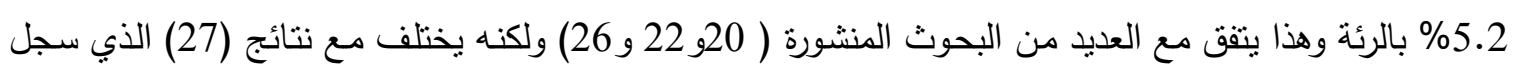

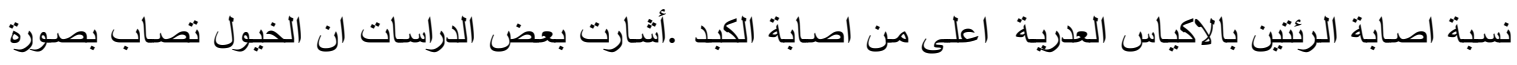

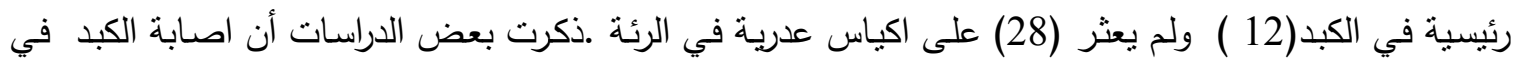

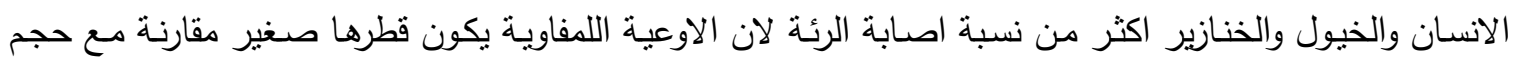
الجنين ( الانسان والخول ولخنازير

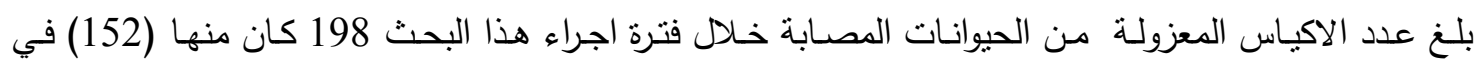

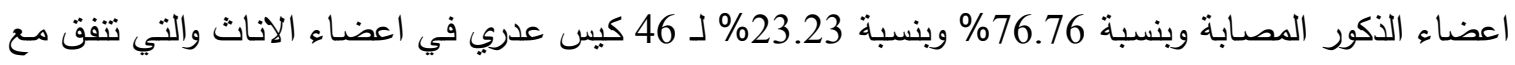

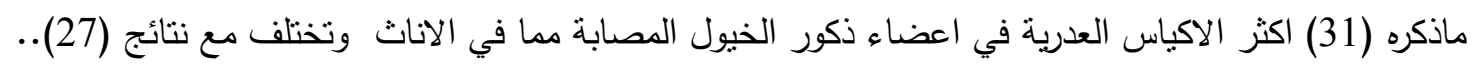

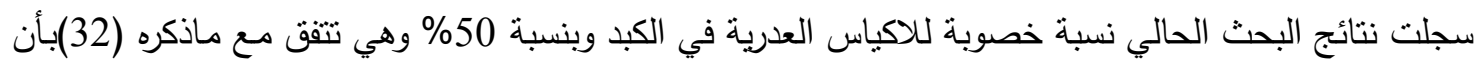

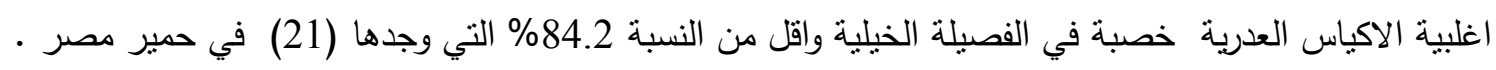


اظهرت النتائج نسبة 16.94\% العقيمة في الكبد وبنسبة 16.88\% في الكبد والرئة معا اما نسبة الاكياس المتكلسة

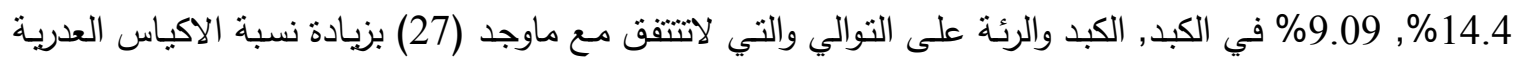
العقيمة عن الاكياس الخصبة من حمير في المغرب.

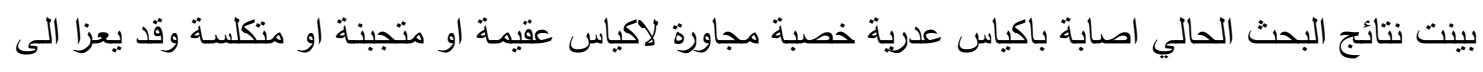

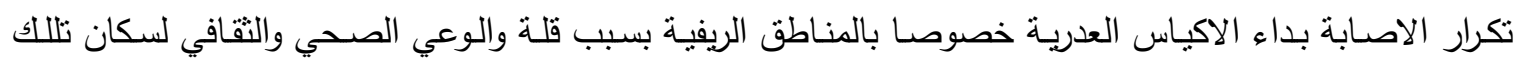

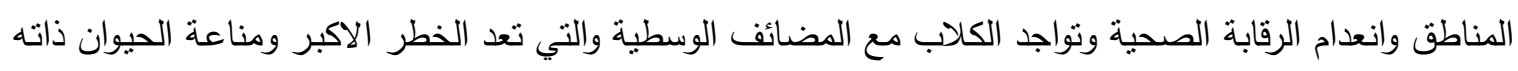

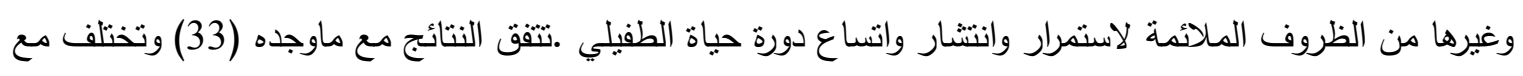

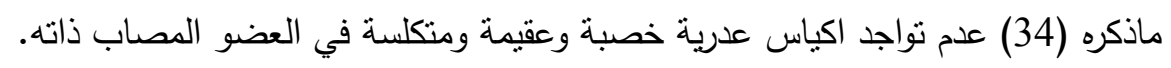

\section{Reference}

1.Anderson Fl (1997) Introduction to cystic echinococosis and description co operative research project in morocco .In :compendium on cystic echinococosis in Africa and middle eastern countries with special reference to morocco (Anderson FL Ouhelli H Kachani ,M.Ed).Brigham young University print services, prove, utach . USA.PP:1-7.

2. Kumaratilake LM Thompson RCA and Eckert J(1986) Echinococus granulosus of equine origin from different countries possess uniform morphological characteristics, Int.J Parasitol . 16:S29-S40.

3. Mukbel RM Torgerson PR and Abo-Shehada MN (2000) Prevalence of hydatidosis among donkeys in North Jordan. Vet parasitol 88:35-42.

4. Lumbery AL (1998) combining data from morphological Trails and genetic markets to determine transmission cycles in the tape worm, Echinococcas granulosus parasitol . 117:185-192.

5. Schantz PM (1999) Echinococcsis. In: Infection disease -principles, pathogenesis and practices (cuerrant $\mathrm{R} \mathrm{L}$ 
Walker D H Walker PF)Churchill living stone Hard count Broce and company. London Toronto Tokyo 10051025.

6. Eckert J Deplazes P Graiy K F Gemmell M A Gottestein D Heath D Jenkins DJ Kamiya $M$ and lightowlers $m$ (2001)_Echinococosis in animals : Clinical aspects, diagnosis and treatment . in :WHO / OIE manual on Echinococosis in humans and animals :public health problem of global concern (Eckert $\mathbf{J}$ Gemmell M Muslin F X Pawlowski Z S.eds )world organization for animal health and world health organization . Paris France: 72-79.

7. Schantz P M Rayn CF Wetty T and Schantz M G(1976) Echinococosis in Arizona and new Mexico Survey of hospital records, 1969-1974 .AM J Trop Hyg 25:312317.

8. Euzeby J A (1999) the epidemiology of hydatidosis with special reference to the mediterrean area parasitol 33:25-39.

9. Bouree P (2001) Hydatidosis dynamics of Transmission world. J. Surg. 25(1):4-9.

10. Smyth J D (1976) Strain difference in Echinococcus granulosus_with special reference to the genus of equine hydatidosis in the united Kingdom. Trans. Roy Soc Trop Med Hyg. 70:93-100

11. Smyth J D (1964) the Biology of hydatid organisms Adv Parasitol. 2:169-219.

12. Lloyd S (2002) personal communication.

13 Hoberg EP Miller S and Brown MA (1994). Echinococus granulosus (taeniidae) and autoch thonous echinococcosis in North American horse. J parasitol. 80:141-144.

14. Ali SR Rahif R H and AL-Kubaisee R Y (1992) prevalence of Internal parasites, among equine in central Iraq . Veterinarian $2: 35-42$. 
15. Daoud M S and AL-Alousi T I (1995) Anote on Some parasites in donkeys in Mosul, Iraq J Vet Scis 8:169-170.

16. Fernandez H (2001) Zoonoses of importance for the economy and for public in out look for yhe elimination of hydatidosis southern. Amer Heath Org Brazil: 2-4

17. Gemmell MA Roberts MG Beard TC and Lawson JR(2001) Quantitative epidemiology and transmission dynamics with special reference to Echinococus granulosus .In WHO/OIE manual on echionococosis in human and animal (Eckert J I femmell MA meslin FX and pawlowski ZS.eds) world organization for animal health and world health organization Paris France. 72-79

18. Carmon C Perdomo R Carob A Alvarez monti J Cranert $R$ Stern D perera G Lloyd S Bazini R Gemmell MA Yarzabal L(1998) Risk factors associated with human cystic echinococosis in Florida, Uruguay : result of amass screening study using ultrasound and serology . AMJ Trop Med Hyg $.58(5): 599-605$.

19. Eckert J Conraths F J Takman K (2000) Echinococosis an emerging or re-emerging zoonosis. Int J parasitol 30:1283-1294. 20. Daily M D and Sweatman G K (1965) the taxonomy of Echinococus granulosus in the donkey and dromedary in Lebanon and syna. Ann Trop Med Parasitol. 59:463-477.

21. Abo-shehada MN (1988) Prevalence of hydatiosis in donkeys from central Jordan. Vet Parasitol. 30:125-130

22. Derbala AA and Zayed AA (1997) prevalence, Fertility and viability of cysticercosis and hydatiosis infections in some domestic animals. J Union Arab Bio. 7(A) zoology: 109-123.

23. Kemmerer WS and Schantz PM (1993) Echinococosis disease. In: infections disease. Clinics of North America. 7(3):605-618.

24. Bowman DD and Lynn RC (1995) parasitology for veterinarians. $6^{\text {th }}$. W. B. Saunders Company. Philadelphia. London Toronto Montreal Sydney Toyo: 137-144.

25. Brown HW and Neva FA (1983) Basic clinical parasitology . $3^{\text {th }}$ ed. Printic Hall. International USA: 191-197. 
26. Ponce-Gordo, F. and Cuesta- Bendera, C.(1997) Differentiation of Spanish strain Echinococus granulosus using larval rostellar hook morphometry. Int. J. parasitol. 27(1):41-49

27. Pandey AM (1980) Hydatidosis. Postgraduate doctor Middle East.3:330-336.

28. Williams RJ and Sweat man GK (1963) on the transmission, biology and morphology of Echinococus granulosus equines anew subspecies of hydatid tape worm in hours in great Britain parasitol . 53:391-407.

29. Heath DD (1971) the migration of oncospheres of Taenia pisiformis, Taenia serials and Echinococus granulosus within the intermediate host Inf.J arasitol.1:145-152.

30. Todorov T and Boeva VC (1999) Human Echinococosis in Bulgaria: A comparative epidemiology analysis. BWHO 77(2):110-116.

31. Edwards GT (1982) Host IgG in equine hydatid cyst fluid .Ann Trop Med Prasitol 76:485-487.

32. Dixon JB Smith JB and Greaore JC (1973) the incidence of hydatid cysts in horses in Great Britain . vet Rec. 93:255.

33. Canley JJ (1979) Equine hydatidosis vet. Rec. 104:411-445.

34. Himonas $\mathrm{C}$ Antoniadou adou-sotriadon $\mathrm{K}$ and Papadopoulos E(1994) hydatidosis of food animals in Greece : Prevalence of cyst containing viable protoscoleces . J Helminthes. 68:311313. 